\title{
Uncertainty Propagation of Internal Heat Gains for Building Thermal Behaviour Assessment: Influence of Spatial Distribution.
}

\author{
Jordan Gauvrit ${ }^{1}$, Antoine Caucheteux ${ }^{1}$, Stéphane Lecoeuche ${ }^{2}$ \\ ${ }^{1}$ Cerema, Equipe-Projet BPE, F-44000 Nantes, France \\ ${ }^{2}$ IMT Lille Douai, Univ. Lille, Unité de Recherche Informatique Automatique, F-59000 Lille, \\ France
}

\begin{abstract}
In building simulation, internal heat gains correspond to heat production by human metabolism or electrical devices use. It is one of the most uncertain model inputs and could have an important impact on building simulation results. This study proposes a method to investigate the influence of the internal heat gains uncertainties by separating the uncertainty on the internal heat gains of the entire building, the uncertainty on the spatial distribution and its evolution on time. The uncertainty sources are propagated independently in a dynamic thermal simulation (DTS). The temperature of each zone at each moment is analyzed. In order to simplify this study, the most representing temperatures are selected with a method based on cumulative variances and a clustering algorithm. This approach is applied on an office building in France. The data coming from a one year monitoring period, provide information to reduce the uncertainties about the real internal heat gains. The results indicate that the effects of the internal heat gains uncertainties are time dependent. They also depend on the heating scenario of the thermal zone (heated or not-heated). At last, the temperatures are mainly influenced by the uncertainty on the internal heat gains of the entire building.
\end{abstract}

\section{Introduction}

Many studies have attempted to reduce the gap between the predicted and measured energy consumption by the calibration of DTS model in the building's monitoring conditions (de Wilde, 2014). Occupant behavior is one of the key parameter in this exercise (Mahdavi \& al, 2009). Indeed, it is necessary to take into account the actions of the occupant on some building components (the settings of Heating Ventilation and Air Conditioning, the opening of windows...) and the internal heat gains due to the occupant body metabolism and the heat provided by the electrical devices. This study will focus on the configuration of internal heat gains for the calibration of DTS.

Data about the internal heat gains of the entire building and its spatial distribution between each zone are needed. For instance, electrical meters may give information about the heat provided by the electrical devices to the entire building. Moreover, a building occupancy schedule can be used to assess the heat gain by human metabolism. These sources of information do not allow to assess precisely the internal heat gains, but various constraints as the cost of the measurement equipment or the respect of privacy limit the availability of more accurate data (Yan et al., 2017; Zhang \& al., 2018). As a results, the internal heat gains of the entire building, the spatial repartition between the thermal zones and the time dependency of spatial repartition still uncertain model parameters. Therefore, the simulated results will be affected by this imprecise setting.

For this reason, uncertainty propagation (UP) techniques have been applied on DTS model to assess the influence of parameters uncertainties on the model outputs. (Ligier \& al. (2017) or Ren \& al. (2017) have assessed the effects related to the uncertainty on the internal heat gains of the entire building. In this work, the uncertainty due to the spatial repartition and its time dependency are also taken into account. The uncertainty sources have been propagated with a Monte-Carlo procedure of $N$ simulations (Saltelli \& al., 2008). (BIPM \& al., 2008) recommends to choose the $N$ number of simulations such as the confidence level of the model output interval reaches a desired value. For a $95 \%$ confidence level, it leads on $N=10^{6}$. However, a DTS required a large computing time and the realization of $10^{6}$ simulations is often not feasible. This paper follows an approximate approach, proposed by the same author: a relatively small value of $N=250$ simulations is performed. The average and the standard deviation of the outputs are computed to characterize the distribution of the model outputs.

The most commonly used outputs in UP, are some global results as the building consumption during one year (Tian et al., 2018), the maximal heating load (Tahmasebi \& al., 2015) or the cumulative distribution of indoor temperature (Mahnameh et al., 2017). Yet, the temperatures of the thermal zones are also interesting outputs of DTS. Recent works such as Maykot \& al. (2018) or Aghniaey et al. (2019) used the measurement of the inside air temperature for a thermal comfort analysis of the building. In an experimental process, the temperature can be a key factor to identify parameters related to the building energy performance (Roels et al., 2017; Ajib, 2018). That is why this contribution focuses on these outputs only.

At the end of the UP, several methods exist to analyze the model outputs variability. The standard deviation and the average of the $N$ observations of each output can be 
computed (Saltelli et al., 2008). However, if the number of output is too large, it will be difficult to summarize these results (Campolongo \& al., 2007).

For this purpose, Campbell \& al. (2006) have reduced the dimension of the outputs space with a principal component analysis. When just a few axis can be retained, this technique allows to interpret easily the UP results. When more axis has to be kept, the interpretation is much more difficult.

\section{Method}

\section{Overview}

In the operation phase, a building monitoring rarely gives a very precise knowledge about the internal heat gains. The lack of information is supposed to be related to:

- The measurement of the total internal heat gains of the building;

- The spatial repartion of the internal heat gains between the thermal zones of the DTS model ;

- The time dependence of spatial repartition.

In order to evaluate the effect of these different uncertainty sources, three UPs are applied to a DTS building model. They take into account independently the uncertainty sources previously mentioned. At the end of one UP, a large number of temperature time series are simulated. A pre-selection step allows to focus the analysis on a part of the outputs whose variances are representative of all the outputs. This step operates in two times:

- First, by finding temporal patterns in the variance of the average temperature of the building; it allows to select shorter periods representative of the entire period of simulation;

- Secondly, the influence of each uncertainty source on the temperature of each thermal zone is assessed by calculating cumulative variances. From these data, the thermal zones are grouped with a clustering algorithm and one thermal zone is selected as a representative of each group.

Finally, the average and the standard deviation of the distribution of the pre-selected outputs are analyzed.

\section{Simulation model}

The model is a multi-zone model. It divides the building into $R$ thermal zones. Each sub-volume is depicted as a uniform temperature and air pressure volume. Each room of the building is considered as a zone of the model. The simulations were performed with (TRNSYS 17, 2010) software and CONTAM software (Dols \& al., 2015) to couple heat and air transfer. These tools were chosen because they allow the prediction of the dynamic of the air temperature of several thermal zones.

During the heated period, a model of an ideal heating system controls each room temperature. This model perfectly adapts the indoor temperature to the temperature set point thanks to a direct acting-control. The building performance monitoring (as described in
'Case study' part) provides information for the parameterization and the definition of its inputs.

The internal heat gains of the model are stored in an internal heat gains matrix $\varphi_{\text {int }}$ defined with:

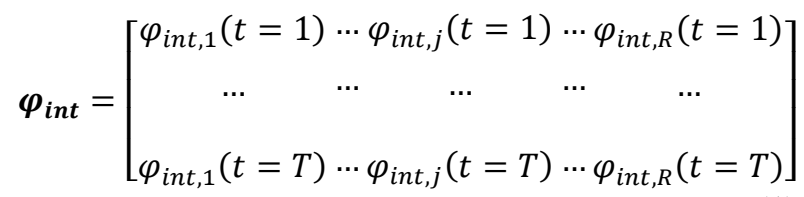

With $t$, the simulation timestep and $T$, the period of simulation. The simulation discretizes a one year period into $T=8760$ hours. $j$ is the index of the modelled thermal zone and $R$ is the number of thermal zones.

The internal heat gain of the entire building, quoted $\varphi_{\text {int }}(t)$ is the sum of the columns of $\boldsymbol{\varphi}_{\text {int }}$.

The part of the internal heat gains assigned to the thermal zone $j$ is called an internal heat gain ratio (noticed $\eta_{j}(t)$ ). In the definition of $\boldsymbol{\varphi}_{\text {int }}$, assumptions have to be made about the time variation of these ratios (fixed ratios or time dependent ratios).

The simulated temperatures are stored in matrix $\boldsymbol{\theta}$ where each element $\theta_{j}(t)$ corresponds to the temperature of the thermal zone $j$ at the time $t$.

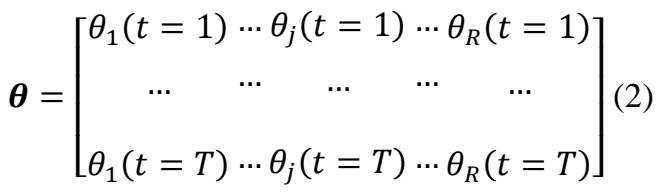

\section{Uncertainty propagation}

First, the probability distribution of the internal heat gains parameters has to be quantified according to the uncertainty source. Rivalin, \& al. (2018) advice to choose normal distribution for measured parameters. For a non-measured parameter, if an expert can suggest a minimum and a maximum value, it will follow a uniform distribution.

The $1^{\text {st }}$ UP is related to the measurement uncertainty of $\varphi_{\text {int }}(t) . \varphi_{\text {int }}(t)$ is estimated with the measurement of the electricity consumption of the entire building and an occupancy schedule built with the building manager. Then, the $i^{\text {th }}$ observation of $\varphi_{\text {int }}(t)$ affected by the measurement uncertainty, is written $\varphi_{\text {int }}{ }^{i}(t)$, and follows:

$$
\varphi_{\text {int }}{ }^{i}(t)=\varphi_{\text {int }}(t) * \varepsilon^{(i)}
$$

with $\varepsilon \sim N(\mu, \sigma)$ where $\mu$ is the mean and $\sigma$ is the standard deviation of a normal distribution. These parameters have been quantified with a measurement uncertainty analysis by considering the uncertainty on the part of electricity consumption reused as a building heat flow, the lack of knowledge about the number of people and their metabolism (Titikpina, 2016). The generation of $N=250$ random draws of $\varepsilon$ is stored in the $1^{\text {st }}$ input sample called $M_{1}$.

The $2^{\text {nd }}$ UP is related to the uncertainty about the spatial repartition under the assumption that internal heat gain ratios are fixed in time. These ratios are defined with 
expert judgment according to the functionality and the available information about the number of occupants in each thermal zone. To take into account the uncertainty on that method, each ratio is supposed to follow $\eta_{j} \sim U\left(a_{j}, b_{j}\right)$ with $a_{j}$ and $b_{j}$ the parameters of a uniform distribution. The generation of $N=250$ random draws of $\boldsymbol{\eta}_{\boldsymbol{j}}$ (the vector of all the fixed ratios) is the $2^{\text {nd }}$ input sample called $M_{2}$.

Finally, the $3^{\text {rd }}$ UP is related to the uncertainty about the spatial repartition under the assumption that internal heat gain ratios are time dependent. However, no known method allows modelling the time evolution of the internal heat gain ratios. It can only be pointed out that some authors proposed stochastic approaches or random walk approaches for modelling the random nature of the occupant presence (Ahn \& al., 2016). In a first approach, a purely random process is used for modelling $\eta_{j}(t)$. Each ratio at time $t$ follows $\eta_{j}(t) \sim U\left(a_{j}, b_{j}\right)$. The uniform distribution parameters are the same as the case of $M_{2}$. The generation of $N=250$ random draws of $\boldsymbol{\eta}_{j}(\boldsymbol{t})$ (the matrix of the random process of all the ratios) is stored in the $3^{\text {rd }}$ input sample $M_{3}$.

The output of the $i^{\text {th }}$ simulation of the UP is noticed $\boldsymbol{\theta}^{\boldsymbol{i}}$. Each element $\theta_{j}^{i}(t)$ of $\boldsymbol{\theta}^{i}$ is the temperature of the thermal zone $j$ at time $t$ for the simulation $i$.

Then, the estimated variance of the response $\theta_{j}(t)$ is noted $V_{j}(t)$ and is computed with:

$$
V_{j}(t)=\frac{1}{N} \sum_{i=1}^{N}\left(E_{j}(t)-\theta_{j}^{i}(t)\right)^{2}
$$

where $E_{j}(t)$ is the estimated average of $\theta_{j}(t)$ which is equal to:

$$
E_{j}(t)=\frac{1}{N} \sum_{i=1}^{N}\left(\theta_{j}^{i}(t)\right)
$$

$V_{j}(t)$ quantifies the variability of each temperature caused by the uncertainty source.

\section{Outputs pre-selection}

First, the average temperature of the building $\theta_{m}(t)$ is computed with:

$$
\theta_{m}(t)=\frac{1}{R} \sum_{j=1}^{R}\left(\theta_{j}(t)\right)(6)
$$

By combining equations (6) and (4), the variance of the average temperature $V_{m}(t)$ is obtained. The variations over the time of $V_{m}(t)$ show the time evolution of the influence of the uncertainties sources. Then, some temporal patterns of $V_{m}(t)$ may exist and are representative of the whole period. Then, the next step will focus on these shorter periods.

The second step is the selection of representative thermal zones. First, the influence of each uncertainty source on each zone $j$ is assessed by the calculation of cumulative variance $C V_{j}$ (Lamboni \& al., 2009) on pre-selected periods :

$$
C V_{j}=\sum_{t_{k}=t_{s}}^{t_{k}=t_{e}} V_{j}(t)
$$

where $t_{s}$ (resp. $t_{e}$ ) is the beginning (resp. the end) of a shorter period. $C V_{j}$ indicates the influence of one uncertainty source on the temperature of the zone $j$ during the period between the interval $\left[t_{s} \ldots t_{e}\right]$. Then, an exponent is added to $C V_{j}$ in order to specify the period and the source of uncertainty.

Thus, each thermal zone is characterized by the cumulative variances computed under the different conditions previously described. The cumulative variances of each thermal zone are stored in the cumulative variances matrix $C$ :

$$
C=\left[\begin{array}{ccc}
C V_{1}^{1} & \cdots & C V_{1}^{S} \\
C V_{2}^{1} & \cdots & C V_{2}^{S} \\
\cdots & \cdots & \cdots \\
C V_{R-1}^{1} & \cdots & C V_{R-1}^{S} \\
C V_{R}^{1} & \cdots & C V_{R}^{S}
\end{array}\right]
$$

Each zone $j$ is associated with the row $j$ of $C$. Each column of $C$ is normalized. The Euclidean distance matrix between each row is computed. Then, a complete linkage method clusters the thermal zones according to a hierarchical scheme. This data analysis was computed with (R Development Core Team, 2018). Finally, for each cluster, one thermal zone is chosen as a representative of the others elements of the same group.

\section{Uncertainty analysis}

The distribution of each pre-selected output is analyzed by representing their confidence interval $(C I)$ with a $95 \%$ confidence level. Under the assumption that $\theta_{j}(t)$ is normally distributed $C I$ follows:

$$
C I=\left[E_{j}(t)-2 \sqrt{V_{j}(t)} ; E_{j}(t)+2 \sqrt{V_{j}(t)}\right](9)
$$

\section{Case study}

The case study is an office building (Figure 1). It was constructed from 2010 to 2012 near to Angers (France). It has two floors. Two parts divide the building according to the usage. One part is the office $\left(853 \mathrm{~m}^{2}\right)$ and the second part is a workshop $\left(703 \mathrm{~m}^{2}\right)$. The building complies with French Thermal Regulation RT2005 that was in effect during its design. However, the performance of the 'office' part is very close to the criteria of the Low Consumption Building - Effinergie label (BBC - Effinergie, 2007). The 'office' part was modelled with TRNSYS software (Titikpina, 2016). The model divides the building into $R=39$ thermal zones that correspond to the same room division of the 'office' part of the building.

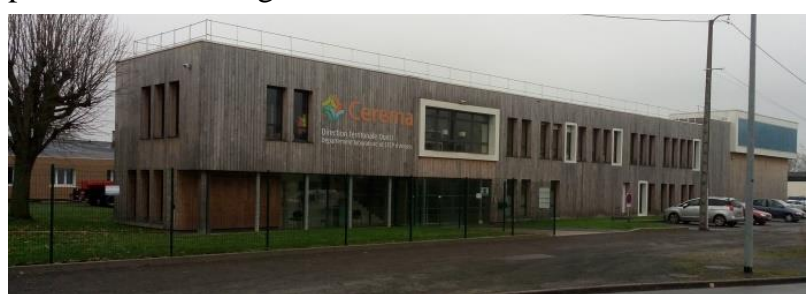

Figure 1: Picture of the case study office building

The simulation computes the building thermal behavior from the $2015 / 03 / 01$ to the $2016 / 02 / 28$. At the end of each simulation, the 39 temperature time series are recorded. During the simulation, a heated period is allocated between 01/03/2015-17/05/2015 and $27 / 09 / 2015-28 / 02 / 2016$. The real not-heated rooms of the building are configured in the model. The local 
weather is monitored. These data are used as inputs of the DTS. Moreover, electricity meters record each hour the building electricity consumption. Combined with the knowledge of an hourly occupancy schedule of the building, these data are used to define the internal heat gains of the entire building $\left(\varphi_{\text {int }}(t)\right)$. The ratios are defined relatively to each another, according to:

- The functionality of the thermal zone: for instance, an office room is fitted with personal computer and office worker can be present in this zone. The ratio will be more important in this room than another room like a corridor;

- The specific knowledge about the real occupancy of the room: for instance, during the monitored period, BUR006 was not occupied. This information was taken into account to affect a lowest ratio to this room.

Titikpina (2016) applies an uncertainty measurement analysis to this case study that shows $\varepsilon \sim N(\mu=$ $1.02, \sigma=0.15)$. The determination of the parameters of the uniform distribution of $\mu_{j}$ is made with an expert judgment seeking to conserve the initial information about the spatial repartition.

\section{Results \& Discussion}

\section{Outputs pre-selection}

Figure 2 shows the variance of the average temperature of the building $\left(V_{m}(t)\right)$. First, it can be noticed that the variance estimated with $M_{1}$ is larger than $M_{2}$ and $M_{3}$. The uncertainty on the entire internal heat gains leads to a larger variability than the uncertainty related to the spatial distribution (with fixed or time varying ratios).

$V_{m}(t)$ follows week variation. It may be caused by the week variation of the entire internal heat gains. On the other hand, it can be noticed that the model response to $M_{1}$ is strongly influenced by the non-heated period. These observations lead to define two shorter periods: « summer» and «winter » as shown in Figure 2.

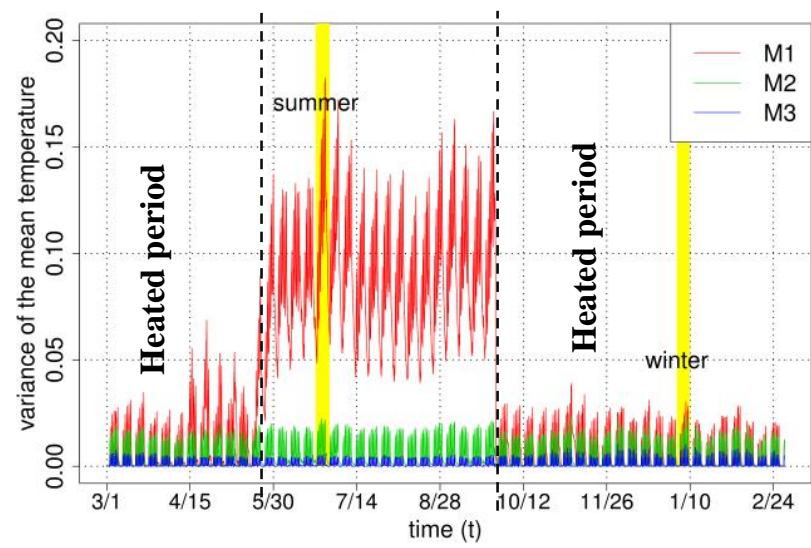

Figure 2: Time evolution of $V_{m}(t)$ according to the uncertainty propagation

$V_{m}(t)$ just gives a global view of the model responses at the building scale. The analysis of the cumulative variances at the thermal zone scale is used to choose representative thermal zone. Figure 3 is a heatmap representation (realized with d3heatmap, a $\mathrm{R}$ package developed by Cheng \& al. (2016)) of the cumulative variances matrix $(C)$. Each row refers to a thermal zone $j$. Each column refers to a particular uncertainty propagation (defined with $M_{1}, M_{2}$ or $M_{3}$ ) and a specific period ('SUM' stands for the summer period and 'WIN' stands for the winter period).

The clustering results are represented on the Figure 3. The rows of $C$ were reorganized in order to position the zones close to the ones with similar cumulative variances.

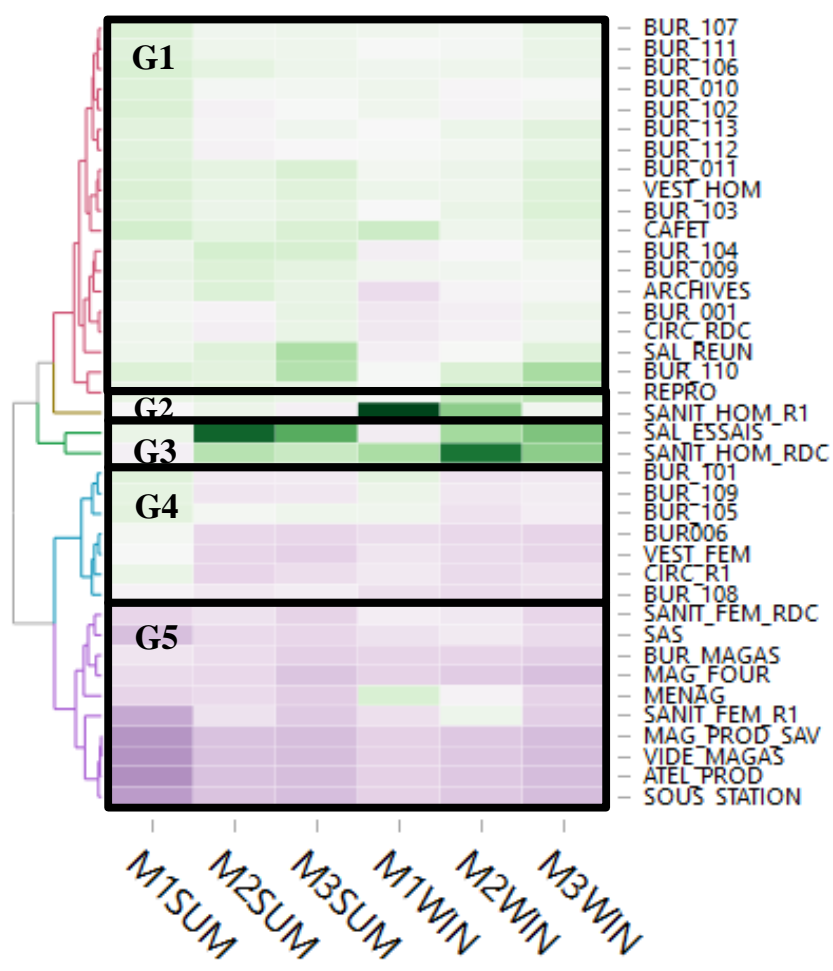

Figure 3: Heatmap and cluster of $C$ - green cells: high level of cumulative variances, purple cells: low level of cumulative variances

It seems relevant to divide the 39 zones into 5 different groups. A representative of each group is arbitrarily selected: BUR_106 for G1, SANIT_HOM_R1 for G2, SAL_ESSAI for G3, BUR_101 for G4, SANIT_FEM_R1 for G5. Figure 4 shows the cumulative variances of each of them. It helps to understand the properties of each group:

- G1 regroups 19 zones. It regroups the most influenced zones by the variation of $M_{1}$ and during the summer period. They are moderately impacted by the others conditions;

- G2 regroups 1 zone : SANIT_HOM_R1. This zone is very affected by the variation of $M_{1}$ and $M_{2}$ during the winter period;

- G3 regroups 2 zones. They are the most influenced zones by the variation of $M_{2}$ and $M_{3}$ during both periods. They are moderately affected by $M_{1}$;

- G4 regroups 7 zones. They are affected by the variation of $M_{1}$ during the summer period. Moreover, they are the less influenced zones by the other 
conditions. Figure 4 shows that the selected thermal zone BUR_101 seems close to the selected thermal zone of G1. There are few distinctive elements between these two objects;

- G5 regroups the last 10 zones. Globally, they are the less impacted zones for all the conditions.

Now, a sufficient number of representative outputs are selected. The next subsection will describe the uncertainty analysis of the chosen ones.

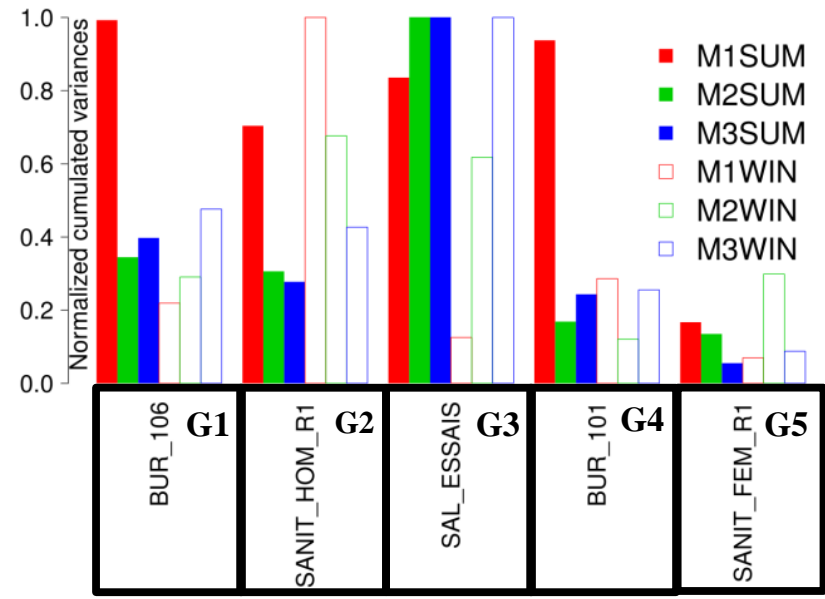

Figure 4 : Normalized cumulative variances according to the selected thermal zones

\section{Uncertainty analysis results}

G1 gathers a majority of thermal zones relied on individual room office (actually, the 'BUR' prefix refers to a room office). Indeed, that kind of rooms is often used at different levels of evaluation of the building thermal performance, and thus, this is a good subject of interest.

BUR_106 (G1) is an individual office $\left(12 \mathrm{~m}^{2}\right)$ on the $1^{\text {st }}$ floor. It is located between two others individual offices and is accessible from the main corridor of the respective floor. This room has a north facing facade. One person occupied this office during the data-monitoring period.

Figure 5, Figure 6 and Figure 7 show the distribution of the temperature of BUR_106 simulated with different input uncertainty sources and during different periods. The black line is the mean value. The grey area covers the confidence interval computed with (9).

Figure 5 shows that the distribution width is affected by the uncertainty of the entire heat gains when the room temperature is not controlled by the heating system. However, during the summer period, the distribution of the temperature around the average value is fixed throughout the all period (Figure 6).

When the influence of the spatial distribution is tested, the distributions are tightening up (Figure 7). The uncertainty related to the distribution has less impact than the uncertainty of the entire heat gains.

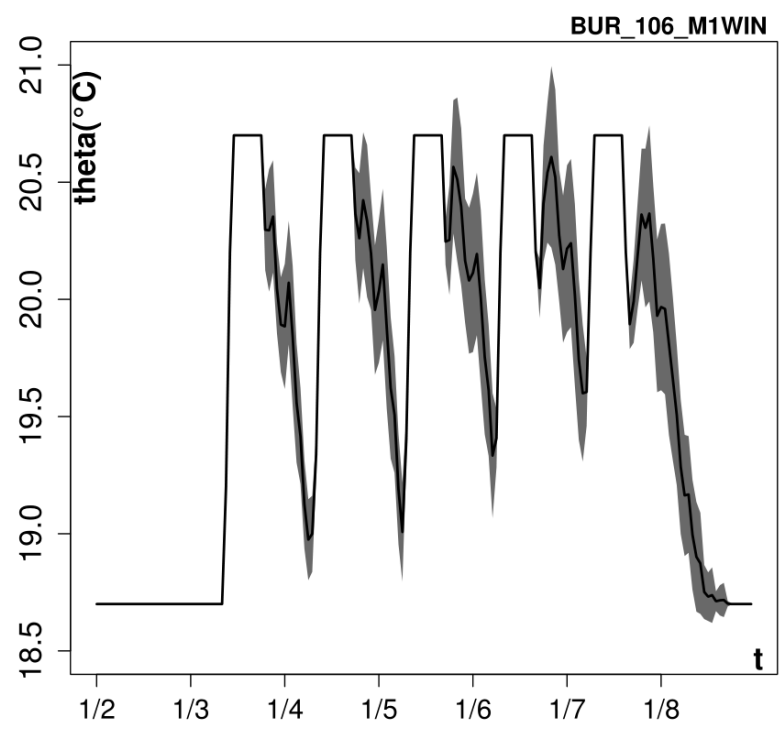

Figure 5: Responses of BUR106 to M1 - Winter period

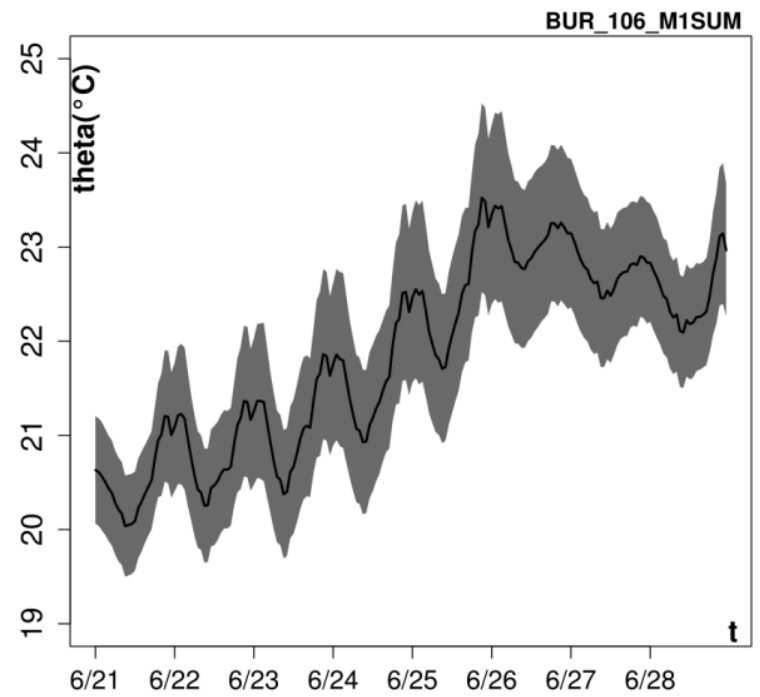

Figure 6: Responses of BUR106 to M1 - Summer period

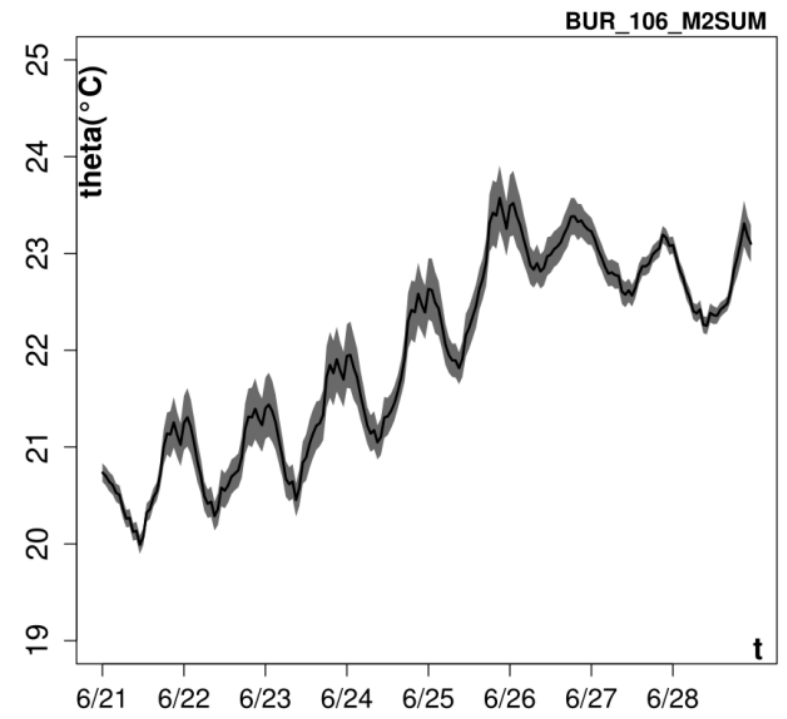

Figure 7: Responses of BUR106 to M2 - Summer period 
The thermal zone SANIT_HOM_R1 belongs to (G2). The distributions are different to BUR_106, especially for the winter period. As this is a non-heated room, the temperature is influenced at each time step of the winter period (Figure 9).

SAL_ESSAI belongs to the group (G3). This zone is identified as the most sensitive zone to the variation of the spatial distribution during the summer period. Figure 8 shows the temperature distribution of this zone obtained with M2. The temperature seems mainly influenced by the spatial repartition during the workdays.

G4 gathers the remaining building offices. The distributions of BUR_101 (G4) are close to the outputs distributions of BUR_106 although the clustering methods lead to two different groups.

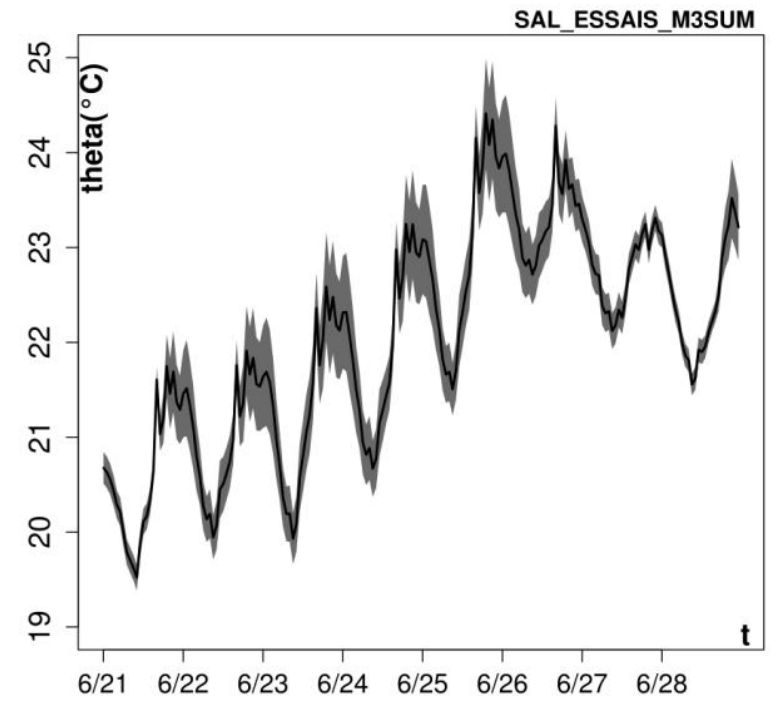

Figure 8 : Responses of SAL_ESSAI to M2 - summer period

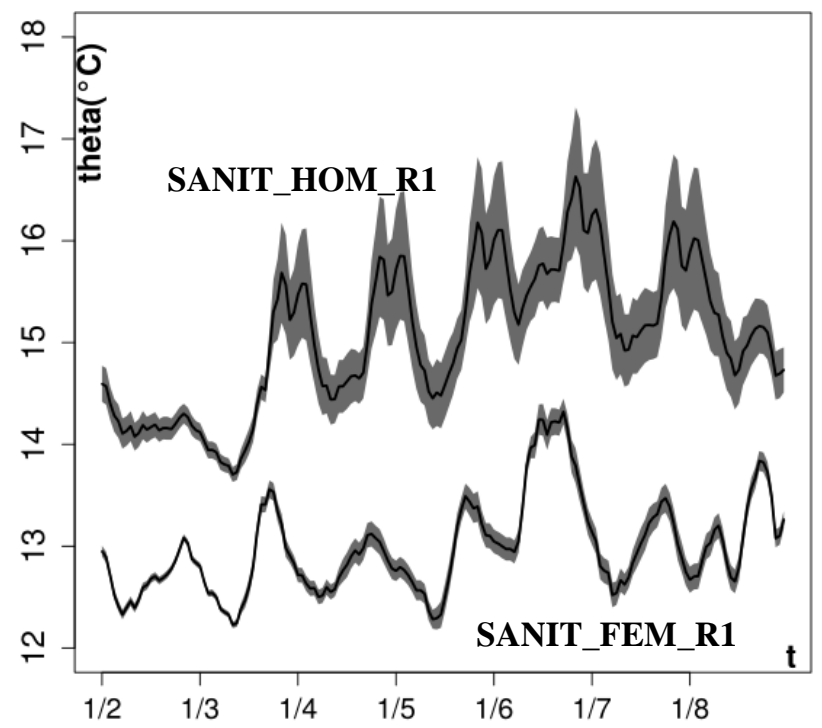

Figure 9: Model responses to M1 the winter period -

Comparison between two non-heated zones

The thermal zone SANIT_FEM_R1 belongs to (G5). As SANIT_HOM_R1, it is a non-heated room too, but the model response is less impacted during the winter period (Figure 9).

\section{Average of standard deviation}

The simulated temperatures could be useful in order to analyze the thermal comfort of every zone of that building. However, the UPs show the simulated temperatures could be sensitive to the accuracy of the internal heat gains. The influence also depends on: the time period, the thermal zone and the uncertainty source. Figure 10 represents the average (in each period) of the standard deviation deducted from the cumulative variances (7).

The highest value of the average of standard deviation is related to the uncertainty on the entire heat gains during the summer period. It is observed for BUR_106 and is equal to $0.4^{\circ} \mathrm{C}$. During the winter period, the hisghest influence is obtained for a non-heated zone (SANIT_HOM_R1) and is equal to $0.2^{\circ} \mathrm{C}$. The accuracy of a thermal comfort analysis based on that DTS could be improved by enhancing the accuracy of the measurement of the entire heat gains.

The uncertainty on spatial distribution could lead on an average of standard deviation equal to $0.2^{\circ} \mathrm{C}$ (SAL_ESSAI during the summer period). However, for the same thermal room and for the same period, in considering that the internal heat gains ratios change randomly in time, the average of standard deviation reaches a value of $0.07^{\circ} \mathrm{C}$.

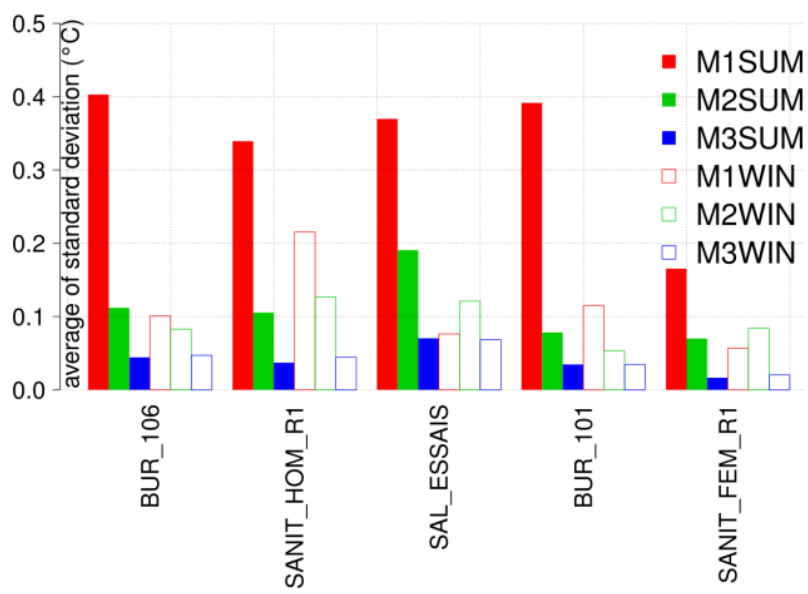

Figure 10 : Average of standard deviation according to the uncertainty sources and the simulated period

\section{Conclusion}

This paper has shown a method to investigate the effect on the building thermal behaviour of different internal heat gains uncertainties (the heat gains of the entire building and their spatial distribution).

Input samples built with the Monte-Carlo approach have been used to estimate the variance of the modelled temperatures of 39 thermal zones over a period of one year. This paper has proposed a two steps method to select the most interesting part of theses outputs.

An office building has been used as a case study. In that case, the uncertainty source that has the most influence was generally the uncertainty on the measurement of th 
entire heat gains. However, the spatial repartition has had a larger influence on the thermal zone temperature with some specific conditions, which are in the presented case: a winter period, a non-heated zone and a spatial repartition fixed in time.

In the case study, the measurement uncertainty of the entire internal heat gains was the most influential parameter. It was mainly influent during the summer period of the simulation. During this period, the average of standard deviation could reach $0.4^{\circ} \mathrm{C}$. It was shown that, if the internal heat gains ratio follows a random process, then, the uncertainty related to the spatial distribution has less impact. This implication can reduce the average of standard deviation from $0.2^{\circ} \mathrm{C}$ to $0.07^{\circ} \mathrm{C}$

The variance of the model outputs depends on the model parameterization and the implementation of the uncertainty propagation. For instance, the cumulative variances of the response are different for a non-heated or a heated zone during the winter period. The identification of more complex features could be a satisfying improvement of this methodology.

To complete this work, the interaction between the entire heat gains uncertainty and the spatial distribution uncertainty should be reviewed. On the other hand, this work could be improved by observing relationships between the input sample factors and the model responses with a sensitivity analysis.

\section{References}

Aghniaey, S., Lawrence, T. M., Sharpton, T. N., Douglass, S. P., Oliver, T., \& Sutter, M. (2019). Thermal comfort evaluation in campus classrooms during room temperature adjustment corresponding to demand response. Building and Environment, 148, 488-497.

Ahn, K.-U., \& Park, C. S. (2016). Different Occupant Modeling Approaches for Building Energy Prediction. Energy Procedia, 88, 721-724.

Ajib, B. (2018). Data-driven building thermal modeling using system identification for hybrid systems. Université de Lille.

BIPM, IFCCC, \& IUAP. (2008). Evaluation of measurement data - Supplement 1 to the "Guide to the expression of uncertainty in measurement" Propagation of distributions using a Monte Carlo method.

Campbell, K., McKay, M. D., \& Williams, B. J. (2006). Sensitivity analysis when model outputs are functions. Reliability Engineering \& System Safety, 91(10-11), 1468-1472.

Campolongo, F., Cariboni, J., \& Saltelli, A. (2007). An effective screening design for sensitivity analysis of large models. Environmental Modelling \& Software, 22(10), 1509-1518.

Cheng, J., \& Galli, T. (2016). d3heatmap: A R package.

de Wilde, P. (2014). The gap between predicted and measured energy performance of buildings: A framework for investigation. Automation in Construction, 41, 40-49.

Dols, W. S., \& Polidoro, B. J. (2015). CONTAM User Guide and Program Documentation Version 3.2 (No. NIST TN 1887).

Hoese, P.-J., Hensen, J. L. M., Loomans, M. G. L. C., de Vries, B., \& Bourgeois, D. (2009). User behavior in whole building simulation. Energy and Buildings, 41(3), 295-302.

Lamboni, M., Makowski, D., Lehuger, S., Gabrielle, B., \& Monod, H. (2009). Multivariate global sensitivity analysis for dynamic crop models. Field Crops Research, 113(3), 312-320.

Ligier, S., Robillart, M., Schalbartt, P., \& Peuportier, B. (2017). Energy Performance Contracting Methodology Based upon Simulation and Measurement. Proceedings from BS2017 Building Simulation Conference. San Francisco (USA), August 2017.

Mahdavi, A., \& Progholf, C. (2009). User Behavior And Energy Performance In Buildings. Proceedings from IEWT 2009 Internationalen Energiewirtschaftstagung an der TU Wien. Vienna (Austria), 2009.

Mahnameh, T., Tahmasebi, F., \& Mahdavi, A. (2017). Consideration of Inhabitants Diversity in Building Performance Simulation: Does It Matter? Proceedings from BS2017 Building Simulation Conference. San Francisco (USA), August 2017.

Maykot, J. K., Rupp, R. F., \& Ghisi, E. (2018). A field study about gender and thermal comfort temperatures in office buildings. Energy and Buildings, 178, 254264.

R Development Core Team. (2018). R: A Language and Environment for Statistical Computing. Retrieved from http://www.R-project.org

Ren, G., Sunikka-Blank, M., \& Zhang, X. (2017). The Influence of the Variation in Occupancy Patterns on Domestic Energy Simulation Prediction: A Case Study in Shanghai. Proceedings from BS2017 Building Simulation Conference. San Francisco (USA), August 2017.

Rivalin, L., Stabat, P., Marchio, D., Caciolo, M., \& Hopquin, F. (2018). A comparison of methods for uncertainty and sensitivity analysis applied to the energy performance of new commercial buildings. Energy and Buildings, 166, 489-504.

Roels, S., Bacher, P., Bauwens, G., Castaño, S., Jiménez, M. J., \& Madsen, H. (2017). On site characterisation of the overall heat loss coefficient: Comparison of different assessment methods by a blind validation exercise on a round robin test box. Energy and Buildings, 153, 179-189.

Saltelli, A., Ratto, R., Andres, T., Campolongo, F., Cariboni, J., Gatelli, D., ... Tarantola, S. (2008). 
Global sensitivity analysis: the primer. West Sussex PO19 8SQ, England: John WIley \& Sons LTd.

Tahmasebi, F., \& Mahdavi, A. (2015). A systematic assement of the sensitivity of building performance simulation results with regard to occupancy related input assumptions. Proceedings from BS2015 Building Simulation Conference. Hyderabad (India), December 2015.

Tatsuo, N., \& Akihiro, N. (2013). Probabilistic approach to determination of internal heat gains in office building for peak load calculations. Proceedings from BS2013 Building Simulation Conference. Chambéry (France), August 2013.

Tian, W., Heo, Y., de Wilde, P., Li, Z., Yan, D., Park, C. S., ... Augenbroe, G. (2018). A review of uncertainty analysis in building energy assessment. Renewable and Sustainable Energy Reviews, 93, 285-301.

Titikpina, F. (2016). Optimisation des plans de mesure dans un contexte de garantie de performance énergétique des bâtiments. Université d'Angers, Angers.

TRNSYS 17 (Version 17). (2010). Retrieved from http://www.trnsys.com/

Yan, D., Hong, T., Dong, B., Mahdavi, A., D’Oca, S., Gaetani, I., \& Feng, X. (2017). IEA EBC Annex 66: Definition and simulation of occupant behavior in buildings. Energy and Buildings, 156, 258-270.

Zhang, Q., Yan, D., An, J., Hong, T., Tian, W., \& Sun, K. (2017). Spatial distribution of internal heat gains: A probabilistic representation and evaluation of its influence on cooling equipment sizing in large office buildings. Energy and Buildings, 139, 407-416.

Zhang, Y., Bai, X., Mills, F. P., \& Pezzey, J. C. V. (2018). Rethinking the role of occupant behavior in building energy performance: A review. Energy and Buildings, 172, 279-294. 